\title{
Heavy Fuel Oil Combustion Characteristics Evaluation in Various Swirling Flow Conditions
}

\section{Xinyan Pei*}

Institute for Aero Engine, Tsinghua University, Beijing, 100084, China peixinyan@mail.tsinghua.edu.cn

\section{A.M. Elbaz}

Clean Combustion Research Center, King Abdullah University of Science and Technology, Thuwal, 239556900, Saudi Arabia Faculty of Engineering Mataria. Helwan University, Cairo, 11795, Egypt ayman.elhagrasy@kaust.edu.sa

\section{Long Jiang}

Clean Combustion Research Center, King Abdullah University of Science and Technology, Thuwal, 239556900, Saudi Arabia long.jiang@kaust.edu.sa

\section{Kamal M. AlAhmadi}

Research and Development Department, Saudi Electricity Company, Thuwal, 23955-6900, Saudi Arabia KkSaeedalahmadi@se.com.sa

\section{Saumitra Saxena}

Clean Combustion Research Center, King Abdullah University of Science and Technology, Thuwal, 23955-6900, Saudi Arabia saumitra.saxena@kaust.edu.sa

William L. Roberts*

Clean Combustion Research Center, King Abdullah University of Science and Technology, Thuwal, 23955-6900, Saudi Arabia william.roberts@kaust.edu.sa

\section{Abstract}

Heavy fuel oil (HFO) is an economical fuel alternative for power generation as its low production cost and high energy density. However, its incomplete combustion induced by the presence of long-chain petroleum molecules in the fuel results in high levels of emissions. Here, we investigate the influence of the swirl flow on the combustion and emissions of a spray HFO swirling flame. To this end, HFO is sprayed into a hot swirling air, using an air-blast nozzle. The flame blowout limits are tested under different swirl flows. An investigation of the in-flame temperature fields, gaseous emissions including $\mathrm{CO}, \mathrm{CO}_{2}, \mathrm{O}_{2}, \mathrm{NO}_{\mathrm{X}}, \mathrm{SO}_{\mathrm{X}}, \mathrm{UHC}$ (Unburned Hydrocarbon) and solid particles in the form of cenospheres are used to quantify the performance of the HFO combustion. The influence of the HFO swirling flame is tested under different conditions of global equivalence ratio, swirling number, and tangential and axial airflow rates. A comparison of two different flame regimes that fuel-jet dominate flame and air-driven vortex flows are investigated and compared in various swirling flow conditions. The results

*Corresponding Author 
40 show that the tangent air is the primary factor for preheating and evaporating the fuel, thus defining the flame 41 operating regimes.

\section{1. Introduction}

The utilization of low-grade fuels (HFO) for power generation and marine transportation is an integral part of the world's increasingly diversifying economy and energy resources due to its low price and high energy density. In general, the price of HFO has been stable at a level of almost $30 \%$ cheaper than the distillate fuel [1]. HFO has a comparable energy density content to distillate fuels [2], but a very high viscosity necessitates extra heating before spray combustion inside a boiler. HFO is also characterized by a high content in asphaltenes, carbon residues, trace metals such as vanadium and nickel, fuel-bound nitrogen and sulfur. Asphaltenes are heavy polycyclic aromatic compounds with embedded heteroatoms; this makes them very difficult to burn, and leads to the formation of large cenospheres, due to an inefficient burning process. The high sulfur content $(>3 \%)$ leads to the formation of harmful $\mathrm{SO}_{\mathrm{x}}$ emissions. The $\mathrm{SO}_{2}$ produced from the combustion of sulfur leads to the formation of $\mathrm{SO}_{3}$ through a subsequent oxidation process. If the exhaust temperatures are lower than the dew point of the acid formed from $\mathrm{SO}_{3}$, cold end corrosion issues can be encountered [3]. Therefore, heavy fuel oil requires more time and heat to achieve full combustion than the lighter distillate oils such as diesel and gaseous fuels (e.g., natural gas, propane).

A concerted effort is needed to continuously improve the efficiency of the combustion and reduce emissions from HFO. To this day, most research on HFO combustion characteristics has been conducted using the singlesuspended droplet $[4,5]$ and multiple free-falling droplet techniques [6, 7]. The main challenges for HFO studies are listed as: a) the complexity of HFO's thermophysical properties, which can vary greatly depending on temperature; b) the inability to mimic the realistic combustion environment of a typical industrial burner at the lab scale. The suspended and falling droplet techniques are adapted to the study of fundamental aspects of HFO's combustion and provide limited information on the characteristics of the spray combustion process in a real furnace. Although the swirling flame is a more suitable technique for HFO combustion, it has been only used in a limited number of studies, which has also not been well-characterized and typically unable to achieve the experimental accuracy required to evaluate the complete combustion performance. turbines [8], engine [9, 10], industrial burners [11], lab-scale burner [12-14] and furnaces [15], for desired flame characteristics. However, there are only specific numbers of studies on HFO's combustion characteristics in a 
of the flame owing through the formation of a recirculation zone and a reduction in combustion length. The high rates of entrainment of the ambient fluid and rapid mixing, predominantly near the boundaries of the recirculation zones, lead to the stable anchoring of the flame. Swirl stabilized flames have been extensively studied in traditional hydrocarbon fuels (e.g., gases, light distillate liquids) at lab-scale. However, there are several studies on the utilization of swirl stabilized flames for highly viscous fuels like HFO or glycerol. Even at lab-scale, the swirlflow burner requires a careful design that enables a robust recirculation zone for fuel with high viscosity. Driscoll and co-workers provided the design and physics-based analysis of lab scale swirl and bluff body burners to study the combustion of methane $[18,19]$. Their swirl burner design was later modified and used by several authors to the combustion of viscous liquid fuels. They highlighted the impacts of the swirl induced vortex circulation and the momentum of the fuel jet on the behavior of the flames. The main feature of the Driscoll burner and its variants used by more recent studies was the ability to control the swirl number, independently of the equivalence ratio. Metzger et al. [20] modified the Driscoll swirl burner design to demonstrate the effective combustion of glycerol combustion for process heating applications with the burner enclosure under $1,000^{\circ} \mathrm{C}$ condition. Myles et al. [21] further modified the design of Metzger's swirl burner to add a metallic interior wall of the combustion chamber for providing the thermal feedback susceptible to warping, due to exposure to high temperatures. Myles et al. [22, 23] used a similar burner for studying the impact of fuel-bound oxygen in oxygenate and hydroxylated fuels on the generation of $\mathrm{NO}_{\mathrm{x}}$.

Based on the Driscoll's design burner, this study focusses on addressing both fundamental and practical aspects of the combustion of HFO to enable a better understanding of HFO's swirling flame properties and the formation of pollutant emissions under various swirling flow conditions. The used burner produces a highly swirl stabilized turbulent HFO jet flame with long residence time to improve the combustion efficiency. In this paper, we provide a detailed description of the swirl-flame experimental setup and the initial set of HFO experiments.

92 We investigate the flame-stabilization map to identify the optimal conditions for a suitable envelope of stable

93 HFO flames. Based on the flame stabilization map, different flames are selected and investigated via studying the

94 in-flame temperature, gaseous and solid particle emissions measurements. We test the performance of the HFO combustion, under various swirling flame conditions, for different global equivalence ratios, swirling numbers, 
98

99

100

101

102

103

104

105

106

107

108

109

110

111

\section{Experimental Methodology}

The HFO sample used in this work is collected from the Shoaiba power plant in Saudi Arabia. Its primary compositional data are analyzed using an Optima 8300 ICP-OES spectrometer (PERKIN-ELMER, INC.) (Table 1). More details about the HFO properties can be found in our previous study [24]. The high content in asphaltenes $(8.2 \%)$ creates a high viscosity of the fuel, up to $530 \mathrm{mPa}$.s at room temperature. Moreover, asphaltenes and carbon residues lead to the formation of the cenosphere and coke during the HFO combustion process. Due to the HFO high sulfur content (3.68\%), the high-temperature exhaust gas is required to prevent the condensation of the acid vapors at the metal surfaces. This, in turn, reduces the overall combustion efficiency. Additionally, trace metals such as vanadium are present in substantial quantities, leading to the hot corrosion of heat transfer surfaces and other components of a typical boiler.

According to the elemental composition shown in Table 1, the fuel global equivalence ratio can be calculated by the mixture global equivalence ratio as $\phi=\frac{(F / O)}{(F / O)_{s t}}$, based on complete combustion under stoichiometric mixture condition.

\begin{tabular}{|c|c|c|c|c|}
\hline \multicolumn{2}{|c|}{$\begin{array}{rr} & \text { Values } \\
\end{array}$} & Units & \multicolumn{2}{|c|}{ LOR $^{\text {a }}$ Test standard } \\
\hline Physical propert & & & & \\
\hline Density $\left(25^{\circ} \mathrm{C}\right)$ & 970 & $\mathrm{~kg} / \mathrm{m}^{3}$ & \multicolumn{2}{|c|}{ ASTM D4052-11 } \\
\hline Viscosity $\left(25^{\circ} \mathrm{C}\right)$ & 530 & mPa.s & \multicolumn{2}{|c|}{ ASTMD445-12 } \\
\hline $\begin{array}{l}\text { Gross calorific } \\
\text { value }\end{array}$ & 42.4 & $\mathrm{MJ} / \mathrm{kg}$ & \multicolumn{2}{|c|}{0.5 ASTM D4868 } \\
\hline $\begin{array}{l}\text { Net calorific } \\
\text { value }\end{array}$ & 40.1 & $\mathrm{~N}$ & \multicolumn{2}{|c|}{0.5 ASTM D4868 } \\
\hline Ash & $<0.1$ & Mass\% & 0.1 & ASTMD482 \\
\hline $\begin{array}{l}\text { Asphaltenes } \\
\text { Elements }\end{array}$ & 8.2 & Mass\% & - & IP 143 \\
\hline Carbo & 84.2 & Mass $\%$ & 0.1 & EPA 440.0 \\
\hline Hydrogen $(\mathrm{H})$ & 11 & Mass\% & & EPA 440.0 \\
\hline Sulphur (S) & 3.68 & Mass\% & \multicolumn{2}{|c|}{ 0.1 ASTM D4294-10 } \\
\hline Nitrogen $(\mathrm{N})$ & 0.2 & Mass\% & 0.1 & EPA 440.0 \\
\hline $\begin{array}{l}\text { Oxygen }(\mathrm{O}) \\
\text { Metal }\end{array}$ & $<5.0$ & Mass\% & 5.0 & EPA 440.0 \\
\hline Vanadium $(\mathrm{V})$ & 38.5 & $\mathrm{mg} / \mathrm{kg}$ & 0.2 & IP 501-05 \\
\hline Nickel(Ni) & 20.7 & $\mathrm{mg} / \mathrm{kg}$ & 0.5 & IP 501-05 \\
\hline Sodium $(\mathrm{N}$ & 24.1 & $\mathrm{mg} / \mathrm{kg}$ & 2.0 & IP 501-05 \\
\hline Zinc & 0.59 & $\mathrm{mg} / \mathrm{kg}$ & 0.5 & \\
\hline & $<5.0$ & $\Pi$ & 5.0 & IP 501- \\
\hline Potassium(K) & $<5.0$ & $\mathrm{mg} / \mathrm{kg}$ & 5.0 & IP 501-05M \\
\hline
\end{tabular}

114 A high-swirl stabilized spray flame chamber is used to combust the HFO efficiently. The configuration of the 115 swirl burner used in the experiments is presented in Fig. 1, which is based on the designs of Driscoll et al. [18, 19, 
116 25]. Detailed descriptions of the subsystems are also illustrated in Fig. 1. These subsystems are listed as a)

117 Insulated combustion chamber subsystem: Due to the difficulty in burning HFO, a side-effect from its high

118 viscosity and high auto-ignition temperature, an insulated combustion chamber with a wall thickness of $7.6 \mathrm{~cm}$ is

119 applied to confine the flame above the nozzle, and a proper warm-up process is necessary. At the start of the

120 procedure, a gaseous propane swirling flame is maintained for 30 minutes to let the combustion chamber to reach

121 a steady temperature, up to $200^{\circ} \mathrm{C}$; b) Fuel supply lines subsystem: The HFO is heated along the fuel line and

122 delivered to the central fuel tube by a syringe pump to control the flow rate with high precision; c) Control and

123 data acquisition subsystem: The radial flame temperature profiles are measured by a thermocouple rake with six

124 R-type thermocouples of $0.25 \mathrm{~mm}$ wire diameter with $\pm 0.25 \%$ tolerance value, spaced $15 \mathrm{~mm}$ apart. This rake of

125 thermocouples is controlled by a linear translation stage with the movement in the radial direction relative to the

126 combustor centerline. The temperature measurement region begins at $35 \mathrm{~mm}$ downstream the tip of the fuel nozzle,

127 due to the diverging exit of the burner, see Fig. 2. This diverging section enhances the flame stability relative to

128 the straight burner exit tip [26]. All thermocouples have been coated and corrected for radiation heat losses.

129 Particulate emissions are filtered and collected from the exhaust gas, using a glass microfiber filter (Whatman ${ }^{\mathrm{TM}}$

130 Binder-Free Glass Microfiber Filters) with the particle retention of $2.7 \mu \mathrm{m}$, which has been widely used in particles

131 collection and analysis under high-temperature conditions [27, 28]. The solid sample captured in the filter is then

132 morphologically analyzed by an FEI ESEM Quanta 600 FEG scanning electron microscope (SEM) and its

133 composition is measured by Energy-dispersive X-ray spectroscopy (EDS);

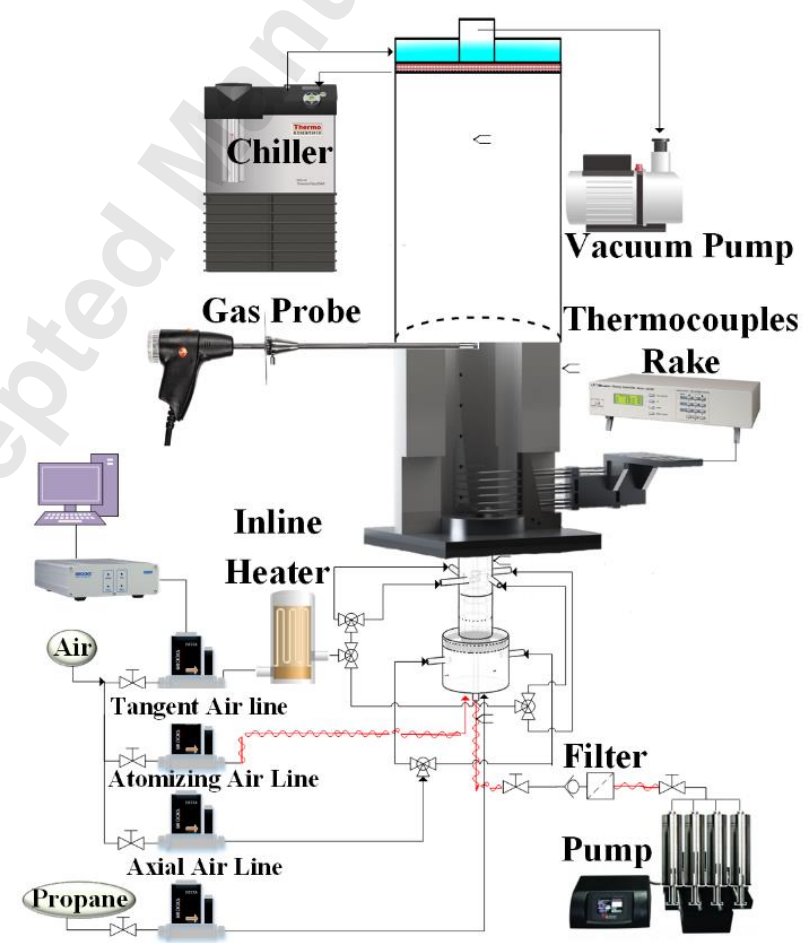

Fig. 1 Experimental setup. 
d) A swirling flow generator subsystem: It is vital to establish an adequate recirculation zone to stabilize the

HFO flame. To this end, a swirl generator is applied in the system, with air entering tangentially and axially to induce a swirling flow pattern. The two air inlets are oriented to control the swirl number. The first air stream is oriented axially and enters through the bottom of the plenum, and its flow rate is designated as $Q_{a}$. Four additional air inlets are oriented tangentially to the burner tube to impart the swirling momentum. The sum of the flow rate of these four inlets is represented as $Q_{t}$. A fuel tube is located axially along the centerline of the burner and extends

143 up through the mixing plenum into a diverging quarl, whereas the exit of the tube is aligned with the base of the diverging quarl. Air flows are controlled using mass flow controllers and heated along the pipes. Characteristics of the swirling jet are usually defined in terms of the swirl number, Sg. The swirl number as given by Syred and Beer[29] is defined as the ratio of the flux of the angular momentum at the throat of the burner to the axial flux momentum times the burner throat radius, thus $\operatorname{Sg}=\int_{d_{f 2}}^{d_{A / 2}}\left(\operatorname{\rho r} U_{a} w_{a}\right) 2 \pi d r /\left[\int_{d_{f 2}}^{d_{A 2}} \rho\left(U_{a}^{2}-W_{a}^{2} / 2\right) 2 \pi d r\left(\frac{d_{A}}{2}\right)\right]$. The aforementioned formula eliminates the need to measure static pressure and allows the use of stereo PIV data to calculate the swirl number[30]. Applying stereo PIV measurements in the current experimental setup is extremely difficult due to the highly sooty of the flame, deposited of oil on the windows, and the mixing of the seeding particles with the HFO, which may lead to high Mie scattered signal (it can damage the CCD camera). However, it was more convenient to track $\mathrm{S}$ by monitoring the tangential and axial mass flow rates of air by determining the geometrical swirl number, Sg. The swirl intensity can be changed by the ratio of tangential and axial airflows, where the geometric swirl number defined by Ribeiro and Whitelaw [30] can be simplified by the equation of $S_{g}=\frac{\left(D_{a}-D_{t}\right) D_{a}}{2 D_{t}^{2}}\left(\frac{Q_{t}}{Q_{t}+Q_{a}}\right)^{2}[31]$; The current burner configuration is relatively simple geometry but replicates many of the fundamental swirling flame behaviors of real gas turbine combustors. In a similar burner but working in gaseous fuel, the actual air swirl number based on the axial and tangent air streams only, without fuel injection, was estimated based on the stereo-PIV measurement at the burner exit. The airflow field measurements at a bulk air velocity of $4.9 \mathrm{~m} / \mathrm{s}$ show a significant reduction in the swirl intensity to 0.32 of the geometrical swirl number,

$160 \mathrm{Sg}$ [26]. This indicates that the actual swirl number is a fraction of the geometrical swirl number and it will be reduced more with the fuel injection.

e) An atomizing fuel system: The most challenging aspect is achieving the proper atomization pattern and droplet sizes for the high viscosity HFO. This requires the atomization system to be capable of producing fine quality atomization of highly viscous liquids. For this purpose, we use a commercially available air atomizing 
166

167

168 representation of the inner structure of the air atomizing nozzle is shown in Fig. 2a, together with the real nozzle and separated parts. The atomizing air is used to spray the fuel through the nozzle; the fuel and air are delivered via a co-annular tube, with the liquid and air streams in the center and outer sections, respectively. At the interface of the nozzle stem, an O-ring seals the liquid channel, and the air stream is separated into 4 channels cutting up the nozzle stem, and below the external nozzle cap. The position of the nozzle in the burner is shown in Fig. 2b.

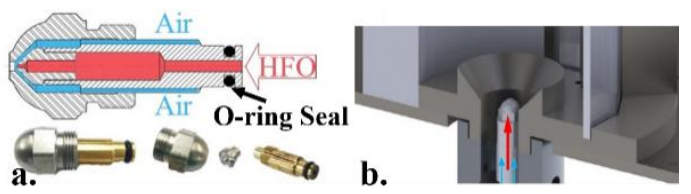

Fig. 2 a. Siphon nozzle; b. Nozzle position.

Once a stable $\mathrm{HFO}$ swirl flame is achieved, we perform measurements of gas-phase emissions on $\mathrm{CO}, \mathrm{SO}_{2}$, $\mathrm{NO}_{x}, \mathrm{UHC}, \mathrm{O}_{2}, \mathrm{CO}_{2}$, from the top of the furnace in the over-fire region with the gas sampling probe shown in Fig. 1. The exhaust gaseous is analyzed using a Testo 350 gas analyzer, which is widely used to evaluate the performance of the liquid fuel combustion [34-36]. Measurements are based on the principle of the ion-selective potentiometer. The analyzer uses a specific gas electrolytic matrix to measure flue gas constituents [37], which is calibrated with standard gas before and after measurements. TESO-350 has an auto-zeroing mechanism that is executed automatically, once the instrument is restarted. Repeatable tests of the experiments on temperature field and gas emission measurements are conducted. In this study, the swirling flame burner is exposed to the atmosphere.

\section{Results and Discussion}

\subsection{Swirling Flame Stability}

The burner used in our study applies a high swirl turbulent spray flame to combust HFO effectively. Different flow parameters can affect the swirling flow pattern like the tangent air and axial airflow rates, and $\mathrm{S}_{\mathrm{g}}$ number. The study of flame stability of HFO's combustion is the first essential step to investigate various boundary conditions and to obtain a qualitative insight into the effects of the swirling flow on the stabilization of the flame.

Fig. 3 presents the measured stability diagram of the HFO's swirl flame, under different $S_{g}$ numbers and bulk air velocities, the bulk air velocity is calculated using the bulk air flow rates at the exit section (including axial air and tangent air). As illustrated in Table 2, the stability limit is examined by gradually increasing the tangent airflow rate from 80 SLPM to 230 SLPM, under three different axial airflow rates of 5 SLPM, 10 SLPM, 15 SLPM, and until the flame is extinguished. This is conducted while keeping the HFO flow rate constant. The air streams are preheated before entering the burner, where the axial and tangent air streams are merged. 
As indicated in Fig. 4, to stabilize the flame under a constant axial airflow rate with increasing the bulk air velocity, it needs to increase the swirling intensity. However, the excessive increase of the swirl intensity

197 destabilize the flame, and unsteady flames are observed by the unstable limits in Fig. 3. The swirling number comes to be higher with a decrease in axial airflow rate from 15 SLPM to 5 SLPM, which providing more residence time for fuel combustion corresponding to higher bulk air velocity of destabilization point increased from $5.7 \mathrm{~m} / \mathrm{s}$ to $6.3 \mathrm{~m} / \mathrm{s}$. In addition, although the effect of the axial air temperature is limited, the difference in the temperature between axial airflow and tangential airflow would amplify the effect of variation in axial airflow on flame stability. When swirl flow is further increased beyond the unsteady points, the flames are blown out.

Table 2 Combustion Stability study conditions

\begin{tabular}{ccc}
\hline \hline & Flow rate & $T /{ }^{\circ} \mathrm{C}$ \\
\hline Tangent air & $80-230$ SLPM & 600 \\
Axial air & $5 / 10 / 15$ SLPM & 300 \\
Atomizing air & $15 \mathrm{SLPM}$ & 150 \\
HFO & $15 \mathrm{ml} / \mathrm{min}$ & 150 \\
\hline \hline
\end{tabular}

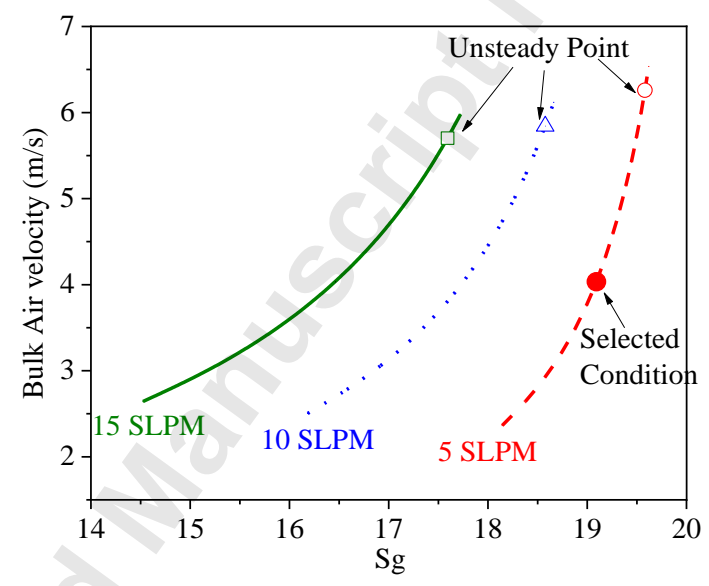

Fig. 3 Stability plot of HFO spray swirling flame at different axial airflow rates.

Based on the stability plot, the stable flame at an axial airflow rate of 5 SLPM is selected for further in-flame measurements highlighted in Fig. 3. The corresponding flow conditions of this selected flame are illustrated in Table 3. The condition of the axial and tangential airflows at 5 SLPM and 150 SLPM respectively with HFO flow rate of $15 \mathrm{ml} / \mathrm{min}$ is selected for stable combustion condition. This results in a global fuel-lean environment of $\phi$ $=0.91$, and $\mathrm{Sg}$ of 19.2 and a power of $9.95 \mathrm{~kW}$. The spray droplet size distribution under non-reacting flow and

211 without swirling flow [38, 39] for this selected flame is given in Fig. 4. The size distribution of fuel droplet spray is measured by a Spraytec spray system from Malvern Instruments. As shown, nearly $80 \%$ of the droplets are

213 under $40 \mu \mathrm{m}$ in diameter. The dominant size of the droplet is approximately $27 \mu \mathrm{m}$ up to $7 \%$ volume frequency. 


\begin{tabular}{lllll}
\hline \hline & Flow rate & $\mathrm{T}$ & $\mathrm{S}_{\mathrm{g}}$ & $\phi$ \\
\hline Tangent air & $150 / \mathrm{SLPM}$ & $600 /{ }^{\circ} \mathrm{C}$ & & \\
Atomizing air & $15 / \mathrm{SLPM}$ & $300 /{ }^{\circ} \mathrm{C}$ & & \\
Axial air & $5 / \mathrm{SLPM}$ & $150 /{ }^{\circ} \mathrm{C}$ & 19.2 & 0.91 \\
$\mathrm{HFO}$ & $15 / \mathrm{ml} / \mathrm{min}$ & $150 /{ }^{\circ} \mathrm{C}$ & & \\
\hline
\end{tabular}

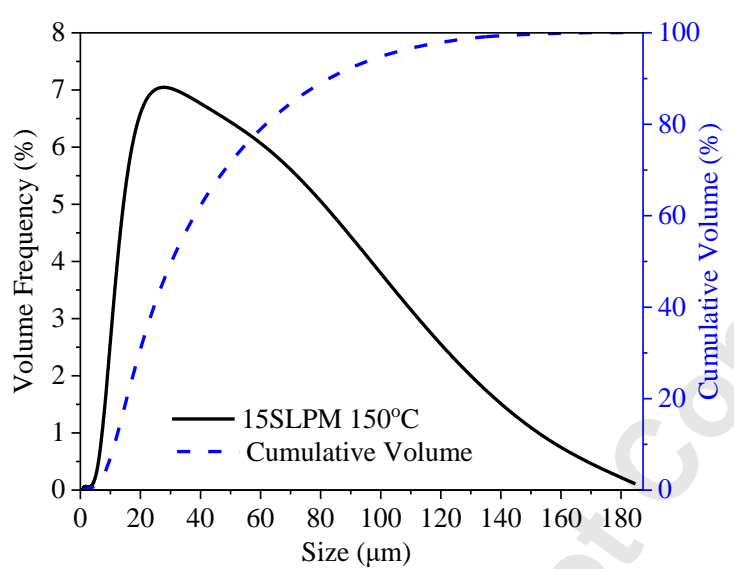

Fig. 4 History of the droplet size of the spray.

The mean centreline flame temperature is given in Fig. 5 with the standard deviation. The temperature profile shows that the most significant difference in temperature is within $1.1 \%\left(12^{\circ} \mathrm{C}\right)$ for the entire range of the flame temperature during the experiment. The peak flame temperature is $1,200^{\circ} \mathrm{C}$ and located at the axial distance equal to $110 \mathrm{~mm}$ on the centerline of the flame within the region of flame temperature measurement. HFO has many hydrocarbons of very high molecular weights ranging from 160 to 100,000 and many unique structures [40]. The centreline flame temperature increases gradually and reaching a peak temperature of nearly $1,100^{\circ} \mathrm{C}$ at an axial distance of $65 \mathrm{~mm}$, which is followed by a small region with a temperature decline.

Further downstream, the temperature again shows a gradual increase. Recently, the TGA analysis on the same HFO sample provided the information that the fuel evaporation occurred in two consecutive steps, where the low boiling gases left out the fuel, while the second phase of the devolatilization occurs at higher temperature [41]. This explained the first flame peak temperature located close to the fuel spray nozzle, which is associated with the first phase of evaporation.

In Fig. 6, the average results of emission measurements with the standard deviation are used to characterize the performance of the HFO's combustion for the selected stable flame. It is worth to mention that the temperature of exhaust gas flue at the sampling location (shown in Fig. 1) is approximately $350^{\circ} \mathrm{C}$. As illustrated in Fig. 6, the 
insignificant compared to the concentration of $\mathrm{CO}_{2}$ (up to more than 12\%). There is only a small amount of UHC left, together with the low percentage of $\mathrm{CO}$ indicate the adequate fuel-air mixing under these flame conditions.

237 Three possible mechanisms can form nitric oxide (NO). The thermal $\mathrm{NO}_{\mathrm{x}}$ significantly occurs when the flame has 238 a high-temperature spot of temperature above $1,500^{\circ} \mathrm{C}$. The prompt formation $\mathrm{NO}_{\mathrm{x}}$ occurs when oxygen is in 239 deficit and high gas temperature and occurs faster than the thermal $\mathrm{NO}_{\mathrm{x}}$, and the oxidation of fuel-bound nitrogen 240 [42]. In the current experiments, the maximum mean temperatures are well below $1,200^{\circ} \mathrm{C}$. Therefore, the 241 measured NO is formed primarily from fuel-bound nitrogen, measured to be $0.2 \%$ mass fraction in the fuel [24], that further oxidizes to nitrogen dioxide $\left(\mathrm{NO}_{2}\right)$. The amount of $\mathrm{NO}_{\mathrm{x}}$ is approximately $280 \mathrm{ppm}$. The emission level

243 of sulfur oxides $\left(\mathrm{SO}_{\mathrm{x}}\right)$ shows a high concentration in the exhaust gaseous, due to the HFO's high sulfur content $244(3.68 \%)$.

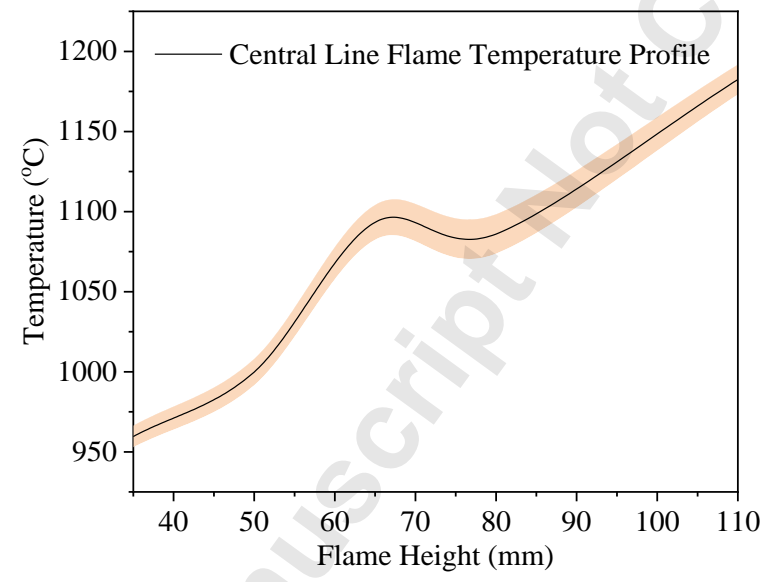

Fig. 5 Central Line Temperature Distribution.

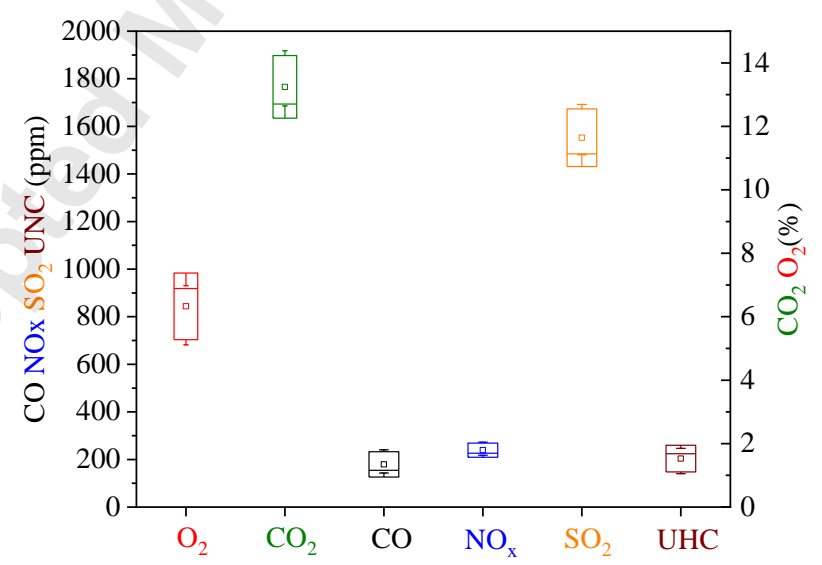

Fig. 6 Gas emission measurement results.

The morphology of the solid particles collected from the flue gas on the exhaust filter is shown in Fig. 7a 
cenospheres. Cenospheres are rigid carbonaceous residues formed by the evolution of the volatile matter that ends abruptly and the collapse of the droplets [43, 44]. The size distribution of cenospheres is presented in Fig. $7 \mathrm{~b}$.

253 Around 37\% (by volume) of the collected particles are in the range of $5 \mu \mathrm{m}$ particle diameter. All over the range 254 of the particles are less than the droplet size of $27 \mu \mathrm{m}$. There is a close relationship between the size of the particles 255 and the diameter of the fuel droplets, which means that the diameters of cenospheres can be controlled by modifying the characteristics of the fuel spray. In Fig. 7c, four specific different morphological points can be distinguished and analyzed in detail. Point 1 is on the surface of a typical cenosphere particle, which has a porous shell structure mainly composed of carbon (up to $90 \%$ ), 5.35\% oxygen, and $5.88 \%$ sulfur. Point 2 is located in the hole on the surface of the same cenosphere, which has the highest concentration in sulfur, up to $18.75 \%$. It is preferable to lock sulfur in the solid emissions rather than in the gaseous ones, for ease of disposal. Point 3 is on the surface of an almost perfectly smooth and spherical particle with relatively high sulfur and oxygen concentrations. This smooth structure contains a minimal amount of silicon and vanadium, both less than $1 \%$. Allouis et al. postulated that the presence of spherical and smooth particles are due to the presence of metallic elements [45]. Point 4 is on the surface of rough particles with flocculent and relatively high oxygen. This morphology of the structure is similar to that observed in low-temperature oxidation-deposition reactions of the fuel [46].

To summarize, in the research on the stability of the swirling HFO flame, HFO contains large amounts of nitrogen and sulfur that form $\mathrm{NO}_{\mathrm{x}}$ and $\mathrm{SO}_{\mathrm{x}}$ during the combustion process, causing undesirable pollution. Although the exhaust contains emissions of cenospheres and gaseous sulfur pollutants, which we try to control and reduce, an HFO spray can be combusted in a swirl burner, efficiently and steady. The results indicate that the size of $80 \%$ of HFO droplets can be controlled within $40 \mu \mathrm{m}$ in diameter under the selected condition at blast air of 15 SLPM, at $150^{\circ} \mathrm{C}$, the axial and tangential airflows at 5 SLPM and 150 SLPM respectively with HFO flow rate of $15 \mathrm{ml} / \mathrm{min}$, resulting in a global fuel-lean environment with $\mathrm{Sg}$ of 19.2 and a power of $9.95 \mathrm{~kW}$. The direct relationship between the size of the particle and the diameter of the fuel droplet means that the diameters of a cenosphere can be controlled and refined by modification of the fuel spray characteristics. The temperature of the flame increases from the fuel nozzle reaching a peak temperature and then is followed with a plateau in temperature, indicating the early combustion of the light species and then followed by another zone in the flame where the heavier species start to burn. 


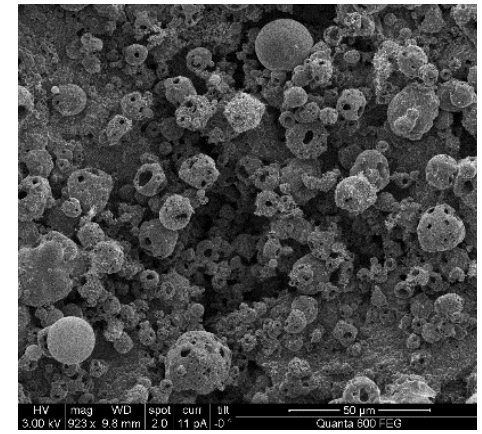

a. SEM morphology.

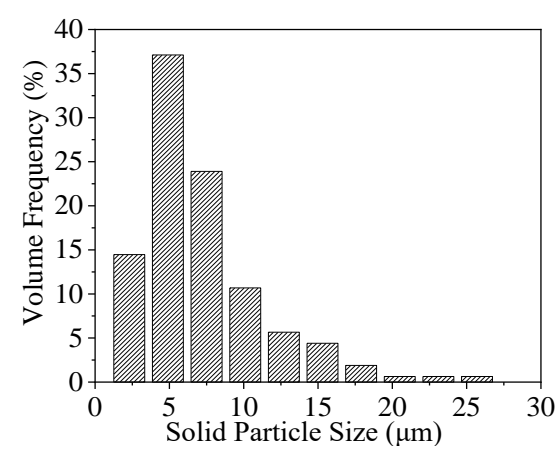

b. Solid particle sizes distribution.

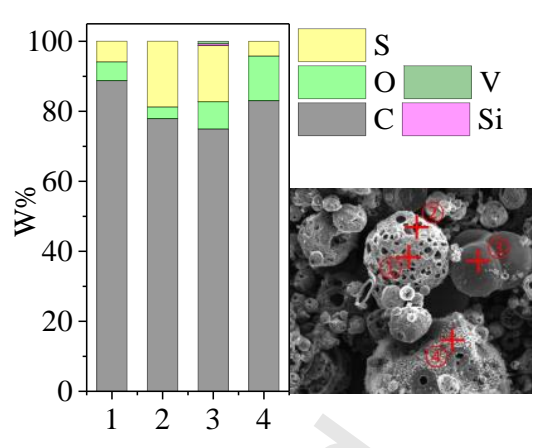

c. Elemental composition

Fig. 7 Characteristics of solid particle emission samples.

\subsection{Effect of Swirling Flow Condition}

In the following section, we will investigate the effects of the swirling flow conditions on the flame structure and emissions. Base on the selected condition in Table 3, the measurements of in-flame temperature distribution and gaseous emissions, including $\mathrm{O}_{2}, \mathrm{CO}_{2}, \mathrm{CO}, \mathrm{SO}_{2} \mathrm{NO}_{x}$, $\mathrm{UHC}$ are conducted under the conditions listed in Table 4 and subdivided into three groups. All the experiments are performed with constant HFO spray characteristics (Fig. 4, at constant HFO and blast airflow rates and temperatures (listed in Table 4). Temperatures of the tangent air and axial air are also kept constant for all cases. In group 1, concerning global equivalence ratios, a fixed Sg number of 19.2 is obtained for Cases 1.1 to 1.3 . This can be achieved by the independent variables in $Q_{a}$ and $Q_{t}$ flow rates with the relatively constant proportion of each flow. Global Equivalence ratios of Case 1.1 and 1.2 are above 1, while the global equivalence ratio of Case 1.3 is below 1. In group 2, the Sg numbers obtained for Cases 2.1 to 2.3 are different. By modifying the proportions of $Q_{t}$ and $Q_{a}$ with a total airflow rate set at 155 SLPM, the obtained Sg numbers range from the low value of 8.5 to high swirl intensity of 19.2 with the same global equivalence ratio of 0.91 . In group 3, the effect of $Q_{a}$ is investigated by keeping the flow rate of the tangent air constant at 150 SLMP, for Case 3.1 to 3.3, but with an axial airflow rate various from 5 SLPM to 15 SLPM.

Table 4 Experimental Conditions

\begin{tabular}{lccll}
\hline \hline & $Q_{t} /$ SLPM & $Q_{a} /$ SLPM & $\mathrm{S}_{\mathrm{g}}$ & $\phi$ \\
\hline Case1.1 & 100 & 3 & & 1.29 \\
Case1.2 & 120 & 4 & 19.2 & 1.1 \\
Case1.3 & 150 & 5 & & 0.91 \\
Case2.1 & 100 & 55 & 8.5 & \\
Case2.2 & 120 & 35 & 12.3 & 0.91 \\
Case2.3 & 150 & 5 & 19.2 & \\
Case3.1 & & 5 & 19.2 & 0.91 \\
Case3.2 & 150 & 10 & 18 & 0.88 \\
Case3.3 & & 15 & 16.9 & 0.86 \\
\hline \hline
\end{tabular}

The flame temperature distribution, for different equivalence ratios, is given in Fig. 8 with the constant Sg of 19.2. In all three cases, we find that the level of temperature increases when the global equivalence ratio 
decreases and the maximum temperature occurs on the centreline, gradually decreasing towards the wall of the

300 combustion chamber. Increasing the tangent airflow rates enhances the flame temperature downstream fuel nozzle,

301 as well as increases the temperature level in the radial direction. This indicates that with increasing the tangent

302 air, more and intense recirculated gaseous increases the rate of fuel evaporation close to the burner exit. According

303 to Driscoll et al. [18], there is an interaction between the fuel jet and air-driven vortex of the swirling flame. For

304 these two types of flames, the fuel-jet dominated flame that forms as a sufficient high fuel-jet to air momentum

305 flux ratio for fuel-jet penetrating the recirculation zone, while the air-driven vortices flame is featured with a

306 strongly recirculating flow. In Fig. 8, the flames of Case 1.1 and 1.2 are similar to fuel-jet dominant flames that

307 fuel jet passes through the centerline, whereas the flow field of Case 1.3 may be under air-driven vortices as the

308 increasing of the recirculation zone.

309 In Fig. 9 and following gas emission profiles, the gaseous emissions concentration is normalized to 15\%

310 oxygen, using the following equation, to exclude the effect of diluted air as $\mathrm{C}\left[@ 15 \% \mathrm{O}_{2}\right]=\mathrm{C}(20.9-15) /(20.9$ -

$311 \mathrm{O}_{2} \%$ ). The uncertainties of the emissions can be referred to Fig. 6 to present the trend clearly. With the increase

312 in the global equivalence ratio, the amount of $\mathrm{NO}_{\mathrm{x}}$ emissions is below $100 \mathrm{ppm}$ in all measurements and decreases

313 by $25.6 \%$ due to the decreased temperatures of the flame. $\mathrm{CO}$ and $\mathrm{UHC}$ increase significantly with the increase in

314 global equivalence ratios from 0.91 to 1.29 due to being off from stoichiometric condition $(\phi=1)$ for the

315 combustion process, resulting in lower combustion efficiency. There are eight times increase in CO profile and

3161.1 times increase in UHC profile from fuel-lean condition (Case 1.3) to fuel-rich condition (Case1.1),

317 respectively. $\mathrm{SO}_{2}$ is found in high levels, at more than $650 \mathrm{ppm}$ for the three cases, with variations within $2 \%$, due

318 to the high concentration of sulfur in HFO. The differences in $\mathrm{CO}_{2}$ and $\mathrm{O}_{2}$ concentrations are relatively small and

319 seem to vary by $5 \%$ maximum.

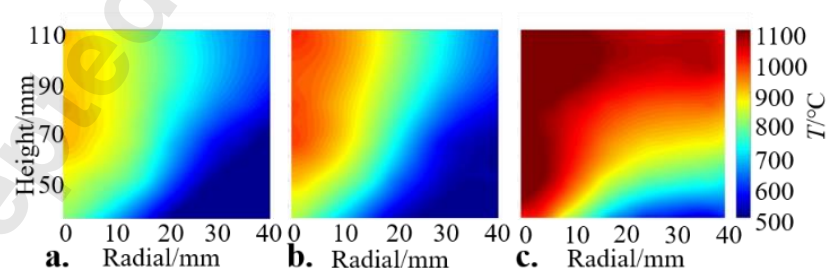

Fig. 8 Flame temperature distribution under global equivalence ratios, a. Case 1.1 $\phi=1.29$; b. Case $1.2 \phi=1.1$; c. Case $1.3 \phi=0.91$. 


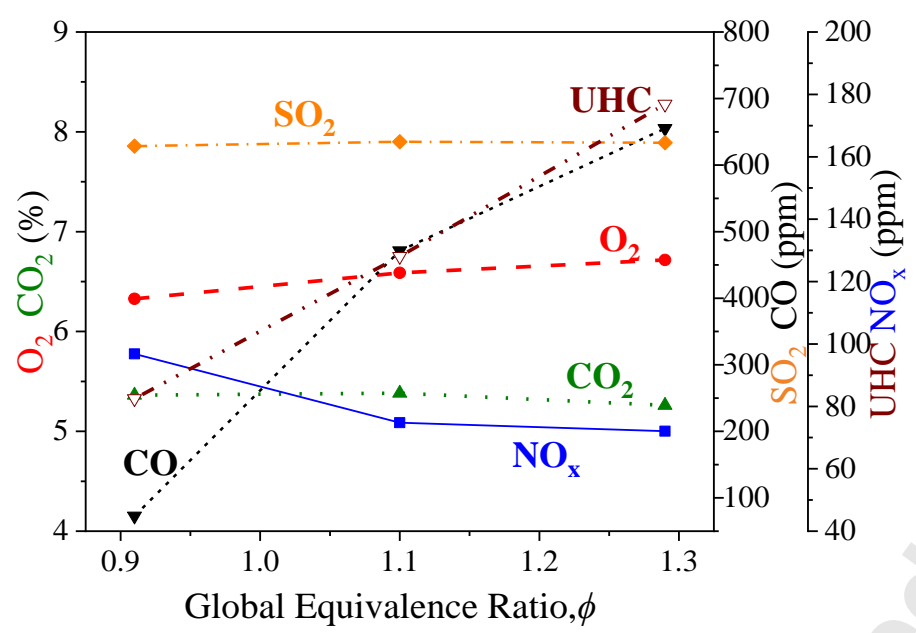

Fig. 9 Gaseous emissions under different global equivalence ratios.

In Fig. 10, the difference in Sg number also affects the temperature distribution of the flame, while keeping the same bulk air velocity and the same thermal input. Distinguished from the temperature profile of Case 2, the flame has a transition from jet-fuel prevailing conditions to a strongly recirculating flame by air-driven vortices in high swirling numbers. Although the total airflow rates are the same in group 2, Case 2.1 and 2.2 may be still under the fuel-jet dominate condition, air-driven vortices control the flame of Case 2.3. Comparing to Case 1.1 and 1.2, we can check that there is a higher temperature distribution in Case 2.1 and 2.2 due to the enhanced recirculation zone with an increase in the axial airflow rate. In Fig. 10a, the rich flame with the high-temperature zone is located at the height of $70 \mathrm{~mm}$ in the $\mathrm{Sg}$ of 8.5 , which moves upstream in the flame direction with the increase in swirling number. Because the air vortex and turbulence intensity of flow increase with a higher swirling number. A higher aromatic content can result in a delayed ignition, and the bigger droplet will need more time to be evaporated. The high Sg number of 19.2 and the tangent airflow rate of 150 SLPM also enhance the combustion in the downstream of the flame, which has a larger high-temperature zone comparing to the two other cases. Therefore, there are two high-temperature zones for the high Sg number of 19.2 with more residence time of the flow: one is the combustion of the lightweight components and small fuel droplet. Downstream of the flame, the high-weight molecular (asphaltene and polycyclic aromatic compounds) and large droplets are preheated and evaporated in the first zone, finally combusting further downstream, at the height of $95 \mathrm{~mm}$ under these particular conditions.

When the swirling number increases, the concentration of gaseous emissions of $\mathrm{O}_{2}, \mathrm{CO}_{2}$, and $\mathrm{SO}_{2}$ in Fig. 11 varies only a little, $4 \%$ maximum. However, $\mathrm{SO}_{2}$ is still at a high level because it is the sulfur content in $\mathrm{HFO}$ that mostly defines the formation of $\mathrm{SO}_{\mathrm{x}}$. As the swirling numbers in group 2 are still very high, the different swirling number has little effect on the oxygen consumption and $\mathrm{SO}_{2}$ content emission under the same global equivalence 
ratio. According to Fig. 11, the formation of $\mathrm{NO}_{\mathrm{x}}$ is highly relevant to the temperature and has a non-linear decrease with a reduction of $6 \%$ at $\mathrm{Sg}$ of 19.1. The decease trend is similar for the profiles of $\mathrm{CO}$ and UHC, with a reduction of 59\% and 34\%, respectively as the Sg number increases. Because stronger recirculating flow in high

351 Sg number enhances the mixture between fuel and air, also improving HFO vaporization performance due to the recirculated hot gaseous towards the early flame region. Moreover, the flame of the lower Sg we observe is weak, unstable and full of noise, in comparison with that observed for a high Sg number, due to an increased release of heat in the axial direction with a high axial airflow rate.

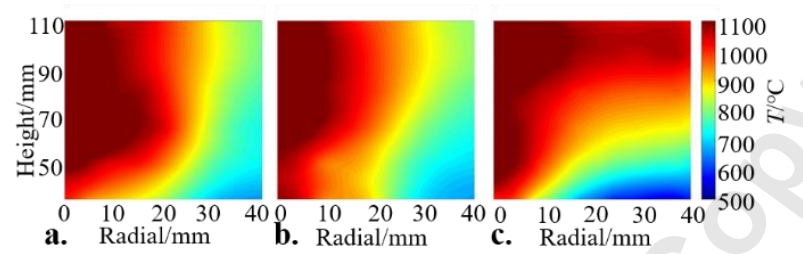

Fig. 10 Flame temperature distribution under the different swirling numbers, a. Case 2.1 Sg=8.5; b. Case 2.2 Sg=12.3; c. Case $2.3 \mathrm{Sg}=19.2$.

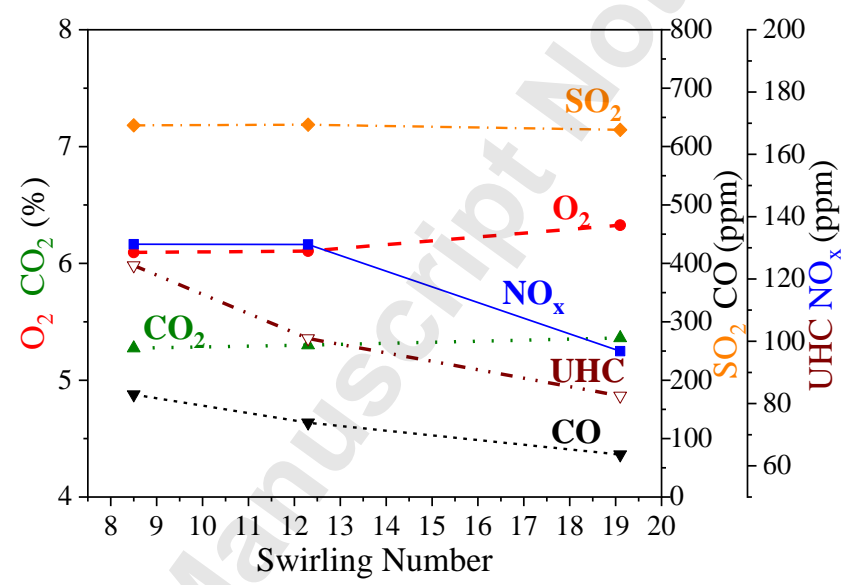

Fig. 11 Gaseous emissions for different swirling numbers.

In Fig. 12, at a fixed flow rate of tangent air, the flames for different axial airflow rates have a similar structure with a wide flame and recirculation zone, corresponding to the air-vortices flame. Increasing axial airflow rates from 5 SLPM to 10 SLPM as indicated in Fig. 12a and b, respectively, lead to change in the temperature distribution within the flames, where a higher level of temperature is recorded further downstream and is radially distributed within the combustor diameter, indicating the enhancement in fuel/air mixing. This indicates a change in the spray recirculated flow interaction. However, the further increase in the axial airflow rates (see Fig. $12 \mathrm{c}$ of the flame with 15 SLPM axial airflow rate) reduces the flame temperature indicating the weaker effect of the recirculation zone in recirculating hot gaseous towards the flame root. This explains the stability map where a higher bulk air velocity requires higher swirl intensity to stabilize the flame. 
As indicated in Fig. 13, the emissions of $\mathrm{CO}_{2}$ and $\mathrm{SO}_{2}$ remain constant with increasing the axial air flow rate.

$\mathrm{CO}$ and UHC decrease by $40.6 \%$ and $63 \%$ respectively, due to a decrease in the global equivalence ratio and the

373 higher swirling intensity. However, the results obtained for $\mathrm{NO}_{\mathrm{x}}$ has an increasing trend of $42 \%$, very differently

374 from the trend of temperature profiles in Fig. 13. The limited temperature diffidence can explain this result of

$375100^{\circ} \mathrm{C}$ and the small fluctuations of the flame under high axial airflow rate conditions.

In brief, in the investigation of different swirling flow conditions, the two different flame regimes of fuel-jet

The maximum flame temperatures increase with increasing tangent air, because more vigorous swirling intensity fuel dominant type to a strongly recirculating flame, due to intensified air-driven vortices in high swirling number.
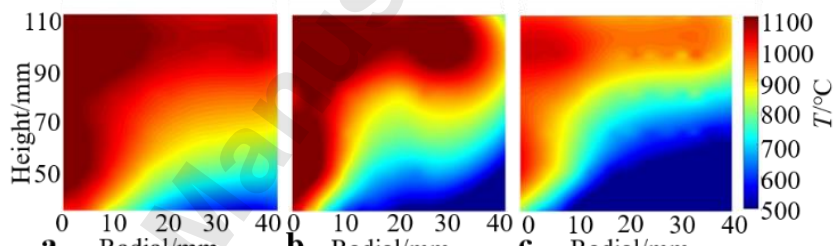

a. $\mathrm{Radial} / \mathrm{mm}$

b. Radial $/ \mathrm{mm}$

c. Radial $/ \mathrm{mm}$

Fig. 12 Flame temperature distribution under different axial air, a. Case 3.1 $Q_{a}=5$ SLPM;b. Case 3.2 $Q_{a}=10$ SLPM; c. Case 3.3 $Q_{a}=15$ SLPM.

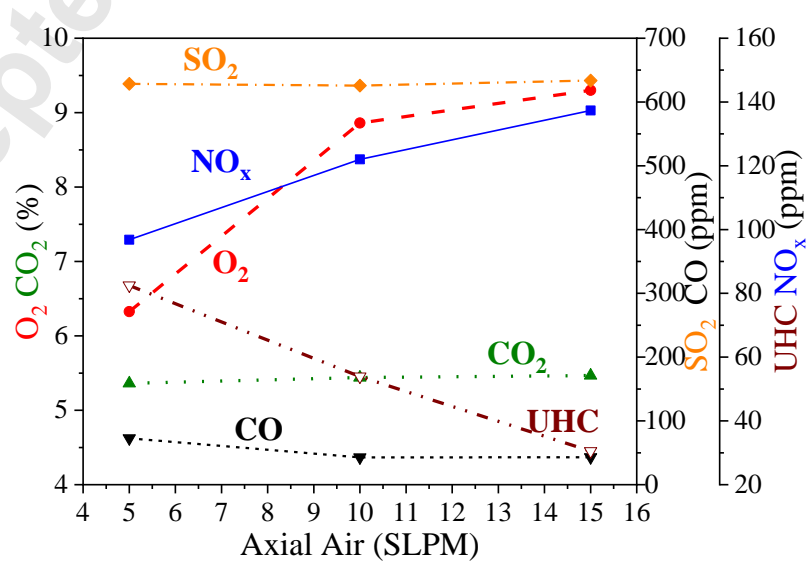

Fig. 13 Temperature profiles and gaseous emissions of different axial air. 


\section{Conclusion}

395

We use a high-swirl stabilized turbulent jet flame burner to efficiently combust HFO, with high residence

396 time and mixture intensity between fuel and air. The stability of the swirling HFO flame and combustion performance are tested with different swirl flow conditions. Although the exhaust contains emissions of cenospheres and gaseous sulfur pollutants, which we try to control and reduce, an HFO spray can be combusted in a swirl burner, efficiently and steady. The direct relationship between the size of the particle and the diameter of the fuel droplet means that the diameters of a cenosphere can be controlled and refined by modification of the fuel spray characteristics. The investigation of various swirling conditions provides a qualitative understanding of the effect of the swirling flow on the HFO combustion characteristics. The parameters of the global equivalence ratio, swirling number, and axial airflow rates are used to characterize the effects of the swirling flow on the flame dynamic in HFO combustion, including temperature distribution, flame regimes, pollutant emissions. The two different flame regimes of fuel-jet dominate flame and air-driven vortex flow are observed and compared by the shapes of the flame temperature distribution caused by the different swirling intensity of the flow. The flame evolves from a jet-fuel dominant type to a strongly recirculating flame, due to intensified air-driven vortices in high swirling numbers. Air-driven vortices flames are characterized by a strong recirculating flow, shorter but more extensive than that observed in fuel-jet flames.

\section{Acknowledgments}

411 The research reported in this publication was supported by Saudi Electricity Company under Grant

412 Agreement number RGC/3/2741-01-01 and by King Abdullah University of Science and Technology (KAUST).

\section{Nomenclature}

$414 \quad C=$ major species concentrations of the gas

$415 D=$ diameter

$416 F=$ fuel

417 HFO $=$ heavy fuel oil

$418 \mathrm{~min}=$ minute

$419 O=$ oxygen

$420 Q=$ air flow rate

$421 r=$ radial distance

$422 \quad$ SLPM $=\quad$ standard liter per minute

$423 T=$ temperature

$424 \quad$ UHC $=$ unburned hydrocarbon

$425 U a=$ average axial air velocity at the burner throat location

$426 W=$ weight

$427 W a=$ azimuthal air velocity components.

$428 \phi=$ global equivalence ratio

$429 \rho=$ air density

$432 a=$ axial direction 
433

434

435

436

437

438

439

440

441

442

443

444

445

446

447

448

449

450

451

452

453

454

455

456

457

458

459

460

461

462

463

464

465

466

467

468

469

470

471

472

473

474

475

476

477

478

479

480

481

482

483

484

485

486

487

488

489

490

st $=$ stoichiometric mixture condition

$t=$ tangential direction

\section{References}

[1] Sun, Z., 2019, "International regulation of heavy fuel oil use by vessels in arctic waters," International Journal of Marine and Coastal Law, 34(3), pp. 513-536.

[2] Czernik, S., and Bridgwater, A. V., 2004, "Overview of applications of biomass fast pyrolysis oil," Energy \& Fuels, 18(2), pp. 590-598.

[3] Ballester, J. M., Fueyo, N., and Dopazo, C., 1996, "Combustion characteristics of heavy oil-water emulsions," Fuel, 75(6), pp. 695-705.

[4] Bartle, K. D., Jones, J. M., Lea-Langton, A. R., Pourkashanian, M., Ross, A. B., Thillaimuthu, J. S., Waller, P. R., and Williams, A., 2013, "The combustion of droplets of high-asphaltene heavy oils," Fuel, 103, pp. 835842.

[5] Villasenor, R., and Garcia, F., 1999, "An experimental study of the effects of asphaltenes on heavy fuel oil droplet combustion," Fuel, 78(8), pp. 933-944.

[6] Urban, D. L., and Dryer, F. L., 1991, "New results on coke formation in the combustion of heavy-fuel droplets," Pro. Combust. Inst., 23(1), pp. 1437-1443.

[7] Ikegami, M., Xu, G., Ikeda, K., Honma, S., Nagaishi, H., Dietrich, D. L., and Takeshita, Y., 2003, "Distinctive combustion stages of single heavy oil droplet under microgravity," Fuel, 82(3), pp. 293-304.

[8] Weigand, P., Meier, W., Duan, X. R., Stricker, W., and Aigner, M., 2006, "Investigations of swirl flames in a gas turbine model combustor: I. Flow field, structures, temperature, and species distributions," Combustion and Flame, 144(1), pp. 205-224.

[9] Bharathi, V. P., and Prasanthi, S. G., 2012, "Experimental investigation on the effect of air swirl on performance and emissions characteristics of a diesel engine fueled with karanja biodiesel," International Journal of Engineering Research and Development, 2(8), pp. 08-13.

[10] Benajes, J., Molina, S., García, J. M., and Riesco, J. M., 2004, "The effect of swirl on combustion and exhaust emissions in heavy-duty diesel engines," Proceedings of the Institution of Mechanical Engineers, Part D: Journal of Automobile Engineering, 218(10), pp. 1141-1148.

[11] Huang, W. C., Hou, S. S., and Lin, T. H., 2017, "Combustion characteristics of a 300kWth oil-fired furnace using castor oil/diesel blended fuels," Fuel, 208, pp. 71-81.

[12] Elbaz, A. M., and Roberts, W. L., 2016, "Investigation of the effects of quarl and initial conditions on swirling non-premixed methane flames: Flow field, temperature, and species distributions," Fuel, 169, pp. 120-134.

[13] Boushaki, T., Merlo, N., Chauveau, C., and Gökalp, I., 2017, "Study of pollutant emissions and dynamics of non-premixed turbulent oxygen enriched flames from a swirl burner," Pro. Combust. Inst., 36(3), pp. 3959-3968. [14] Shi, B., Cao, Q., Xie, D., Peng, W., and Wang, N., 2018, "A novel combustion system for liquid fuel evaporating and burning," Pro. Combust. Inst., 37, pp. 4329-4336.

[15] Zhou, H., Ren, T., and Yang, Y., 2015, "Impact of a on combustion and NOx emissions of a large-scale laboratory furnace fired by a heavy-oil swirl burner," Appl. Therm. Eng., 90, pp. 994-1006.

[16] Pei, X. Y., Abdul Jameel, A. G., Chen, C., AlGhamdi, I. A., AlAhmadi, K. M., AlBarakati, E., Saxena, S., and Roberts, W. L., 2020, "Swirling flame combustion of heavy fuel oil: Effect of fuel sulfur content," Journal of Energy Resources Technology, pp. 1-16.

[17] Zhou, H., Ren, T., Huang, Y., Hu, S., and Cen, K., 2013, "Low-NOx modification of a heavy fuel oil swirl burner based on semi-industrial scale experimental tests," Energy \& Fuels, 27(9), pp. 5029-5035.

[18] Chen, R.H., Driscoll, J. F., Kelly, J., Namazian, M., and Schefer, R. W., 1990, "A comparison of bluff-body and swirl-stabilized flames," Combust. Sci. Technol., 71(4-6), pp. 197-217.

[19] Feikema, D., Chen, R.H., and Driscoll, J. F., 1990, "Enhancement of flame blowout limits by the use of swirl," Combustion and Flame, 80(2), pp. 183-195.

[20] Metzger, B., 2007, "Glycerol combustion," MSc Thesis MSc Thesis, North Carolina State University, Raleigh, NC, USA.

[21] Bohon, M. D., Metzger, B. A., Linak, W. P., King, C. J., and Roberts, W. L., 2011, "Glycerol combustion and emissions," Pro. Combust. Inst., 33(2), pp. 2717-2724.

[22] Bohon, M. D., Al Rashidi, M. J., Sarathy, S. M., and Roberts, W. L., 2015, "Experiments and simulations of nox formation in the combustion of hydroxylated fuels," Combustion and Flame, 162(6), pp. 2322-2336.

[23] Bohon, M. D., and Roberts, W. L., 2013, "NOx emissions from high swirl turbulent spray flames with highly oxygenated fuels," Pro. Combust. Inst., 34(1), pp. 1705-1712.

[24] Elbaz, A. M., Gani, A., Hourani, N., Emwas, A. H., Sarathy, S. M., and Roberts, W. L., 2015, "TG/DTG, FT-ICR mass spectrometry, and NMR spectroscopy study of heavy fuel oil," Energy \& Fuels, 29(12), pp. 78257835 . 
491

492

493

494

495

496

497

498

499

500

501

502

503

504

505

506

507

508

509

510

511

512

513

514

515

516

517

518

519

520

521

522

523

524

525

526

527

528

529

530

531

532

533

534

535

536

537

538

539

540

541
[25] Feikema, D., Chen, R.-H., and Driscoll, J. F., 1991, "Blowout of nonpremixed flames: Maximum coaxial air velocities achievable, with and without swirl," Combustion and Flame, 86(4), pp. 347-358.

[26] Elbaz, A. M., Yu, S., Liu, X., Bai, X. S., Khesho, I., and Roberts, W. L., 2019, "An experimental/numerical investigation of the role of the quarl in enhancing the blowout limits of swirl-stabilized turbulent non-premixed flames," Fuel, 236, pp. 1226-1242.

[27] Borrás, E., Tortajada-Genaro, L. A., Vázquez, M., and Zielinska, B., 2009, "Polycyclic aromatic hydrocarbon exhaust emissions from different reformulated diesel fuels and engine operating conditions," Atmospheric Environment, 43(37), pp. 5944-5952.

[28] Irimiea, C., Faccinetto, A., Carpentier, Y., Ortega, I.-K., Nuns, N., Therssen, E., Desgroux, P., and Focsa, C., 2018, "A comprehensive protocol for chemical analysis of flame combustion emissions by secondary ion mass spectrometry," Rapid Communications in Mass Spectrometry, 32(13), pp. 1015-1025.

[29] Syred, N., and Beér, J. M., 1974, "Combustion in swirling flows: A review," Combustion and Flame, 23(2), pp. 143-201.

[30] Ribeiro, M. M., and Whitelaw, J. H., 1980, "Coaxial jets with and without swirl," Journal of Fluid Mechanics, 96(4), pp. 769-795.

[31] Zhen, H. S., Leung, C. W., and Cheung, C. S., 2009, "Heat transfer from a turbulent swirling inverse diffusion flame to a flat surface," Int. J. Heat Mass., 52(11), pp. 2740-2748.

[32] Sequera, D., Agrawal, A. K., Spear, S. K., and Daly, D. T., 2008, "Combustion performance of liquid biofuels in a swirl-stabilized burner," J. Eng. Gas Turbines Power, 130(3), pp. 032810-032819.

[33] Jun, H. M., Kim, J. H., Lee, S. H., and Yoh, J. J., 2018, "Towards simplified monitoring of instantaneous fuel concentration in both liquid and gas fueled flames using a combustor injectable libs plug," Energy, 160, pp. 225-232.

[34] Koc, A. B., and Abdullah, M., 2013, "Performance and NOx emissions of a diesel engine fueled with biodiesel-diesel-water nanoemulsions," Fuel Processing Technology, 109, pp. 70-77.

[35] Azoumah, Y., Blin, J., and Daho, T., 2009, "Exergy efficiency applied for the performance optimization of a direct injection compression ignition (CI) engine using biofuels," Renewable Energy, 34(6), pp. 1494-1500.

[36] Çelikten, İ., Mutlu, E., and Solmaz, H., 2012, "Variation of performance and emission characteristics of a diesel engine fueled with diesel, rapeseed oil and hazelnut oil methyl ester blends," Renewable Energy, 48, pp. 122-126.

[37] Testo350, 2017, "Combustion \& emission analyzer instruction manual,."

[38] Triballier, K., Dumouchel, C., and Cousin, J., 2003, "A technical study on the spraytec performances: Influence of multiple light scattering and multi-modal drop-size distribution measurements," Experiments in Fluids, 35(4), pp. 347-356.

[39] Priol, L., Baudel, P., Louste, C., and Romat, H., 2006, "Theoretical and experimental study (linear stability and malvern granulometry) on electrified jets of diesel oil in atomization regime," Journal of Electrostatics, 64(7), pp. 591-596.

[40] Baert, R. S. G., 1993, "A mathematical model for heavy fuel droplet vaporization and pyrolysis in a high temperature inert gas," Combust. Sci. Technol., 90(1-4), pp. 125-147.

[41] Khateeb, A., Elbaz, A. M., Guida, P., and Roberts, W. L., 2018, "Influence of asphaltene concentration on the combustion of a heavy fuel oil droplet," Energy \& Fuels.

[42] Li, Y. H., Radovic, L. R., Lu, G. Q., and Rudolph, V., 1999, "A new kinetic model for the no-carbon reaction," Chem. Eng. Sci., 54(19), pp. 4125-4136.

[43] De Blas, L. J. M., 1998, "Pollutant formation and interaction in the combustion of heavy liquid fuels," $\mathrm{PhD}$ thesis PhD thesis, University of London, London, England.

[44] Elbaz, A. M., khateeb, A. A., and Roberts, W. L., 2018, "Pm from the combustion of heavy fuel oils," Energy, 152, pp. 455-465.

[45] Allouis, C., Beretta, F., and D’Alessio, A., 2003, "Structure of inorganic and carbonaceous particles emitted from heavy oil combustion," Chemosphere, 51(10), pp. 1091-1096.

[46] Pei, X. Y., and Hou, L. Y., 2016, "Effect of dissolved oxygen concentration on coke deposition of kerosene," Fuel Processing Technology, 142, pp. 86-91. 